\title{
Shape and Thickness of Cushion in a Standing Aid to Support a Forward Bending Posture: Effects on Posture, Muscle Activities and Subjective Discomfort
}

\author{
Kazuyuki IWAKIRI ${ }^{1 *}$, Midori SOTOYAMA ${ }^{1}$, Ippei MORI ${ }^{1}$, Hiroshi JONAI ${ }^{2}$ and Susumu SAITO ${ }^{1}$ \\ ${ }^{1}$ National Institute of Industrial Health, 21-1, Nagao 6-chome, Tama-ku, Kawasaki 214-8585, Japan \\ ${ }^{2}$ Nihon University, 1-8-14, Kandasurugadai, Chiyoda-ku, Tokyo 101-8308, Japan
}

Received December 11, 2002 and accepted October 8, 2003

\begin{abstract}
In order to prevent low back pain (LBP) during dishwashing, we developed three types of aid with a thick cushion for supporting the shins and evaluated the effects of the standing aid on the subjective discomfort and muscle activities. Nine female volunteers were asked to wash plates in each of four working postures: (a) without the standing aid, (b) with the round type of aid, (c) with the small rectangular type of aid, and (d) with the large rectangular type of aid. With the three types of aid, the subjects were instructed to support the shins with the standing aid and to support the abdomen with the edge of a kitchen counter. In the three postures with the standing aid, $21.5 \pm$ $10.0 \%$ of the weight was supported with the standing aid and the kitchen counter. The subjective discomfort was milder and the muscle activity level in the low back was lower in the three postures with the standing aid than in the posture without the aid. It was thought that the round type of aid would be more effective in decreasing the discomfort in many of body regions and the muscle load on the low back than either of the rectangular types of aid. Therefore, it was suggested that the standing aid had the desired effect in decreasing discomfort and muscle load on the low back during dishwashing.
\end{abstract}

Key words: Low back pain (LBP), Standing aid, Forward bending posture, Subjective discomfort, Muscle activity, Dishwashing

\section{Introduction}

The number of low back pain (LBP) cases accounts for approximately $60 \%$ of all occupational diseases and totaled approximately 4,700 in $2000^{1}$. In facilities for providing meals, LBP among cooks has become a main problem in occupational health ${ }^{2-10)}$. Cleaning tasks in the facilities, such as washing of cookware and tableware, increases the workload on the low back ${ }^{6,10)}$. In washing, the worker must keep the trunk bent forward for long periods ${ }^{9)}$, because a large deep sink is used in washing dishes. The long time forward bending posture is indicated as one of the risk factors for $\mathrm{LBP}^{11,12)}$.

*To whom correspondence should be addressed.
The guidelines for the prevention of LBP in workplaces indicate the measures for LBP prevention ${ }^{12)}$. In the guidelines, it is indicated that excessive workload on the low back should be reduced by whole or partial automation or mechanization of the work, or if that is difficult, that adequate supplemental equipment should be introduced for workload reduction. For dishwashing, an automatic washing machine is used at present in most facilities providing meals. Before using the machine, the worker needs to wash dishes with the hands to remove leftovers, which is called preliminary washing. There is no suitable supplemental equipment for LBP prevention in preliminary washing.

We developed supplemental equipment, a standing aid, to support the forward bending posture during preliminary washing and evaluated the effects of the aid in decreasing 
the loads on the low back and the legs in our previous study ${ }^{13)}$. Subjects leaned on the standing aid and the edge of a kitchen counter. In that posture, the standing aid and the edge of the kitchen counter supported the shins and the abdomen, respectively. It was found that the standing aid decreased the muscle loads on the low back and legs, but did not decrease the subjective discomfort in the low back. In the previous study, the cushion of the standing aid was thin and the shape of the cushion was a small rectangle. Since the thickness of the cushion would change the force when leaning on the standing aid and the shape of the cushion would change the leaning posture, both these factors in the cushion might help to decrease the workload on the low back. Therefore, we thought to examine the effect of a thick cushion and other types of cushion on low back discomfort. In this study, we developed three types of aid with a thick cushion for supporting the shins and evaluated the effects of the new aid on the subjective discomfort and the muscle activities.

\section{Methods}

\section{Subjects}

Nine female volunteers were asked to participate in this experiment. Ages ranged from 21 to $28 \mathrm{yr}$ (mean $=24.8 \mathrm{yr}$, standard deviation: $\mathrm{SD}=2.3 \mathrm{yr}$ ). Mean height with $\mathrm{SD}$ was $158.8 \pm 4.7 \mathrm{~cm}$ and mean body weight with SD was $53.5 \pm 6.9 \mathrm{~kg}$. No subject had a history of acute or chronic LBP. Each subject wore a shirt, slacks and sandals during the experiment. The objectives and method of this study were explained to the subjects, and written informed consent was obtained from them before the experiment.

\section{Standing aid and kitchen utensil}

Figure 1 shows a newly developed standing aid with a round type of cushion and a kitchen counter. The standing aid consisted of an iron board (width $700 \mathrm{~mm} \times$ length 450 $\mathrm{mm} \times$ height $10 \mathrm{~mm}$ ), a steel prop (width $32 \mathrm{~mm} \times$ length $32 \mathrm{~mm} \times$ height $850 \mathrm{~mm}$ ), a steel bar column (diameter 18 $\mathrm{mm} \times$ length $500 \mathrm{~mm}$ ) and a support cushion. Three types of support cushion were made of sponge with a thickness of $50 \mathrm{~mm}$ : round type (diameter $120 \mathrm{~mm} \times$ length $400 \mathrm{~mm}$ ), small rectangular type (width $400 \mathrm{~mm} \times$ length $200 \mathrm{~mm}$ ) and large rectangular type (width $400 \mathrm{~mm} \times$ length $300 \mathrm{~mm}$ ). The round type of cushion was made by winding the sponge around a bar with a diameter of $20 \mathrm{~mm}$. The sponge of the cushion was made of flexible polyurethane foam and was covered with synthetic leather. The sponge was $30 \mathrm{~mm}$ thicker than that in our previous experiment ${ }^{13)}$ and the elasticity was same. The height of the cushion could be

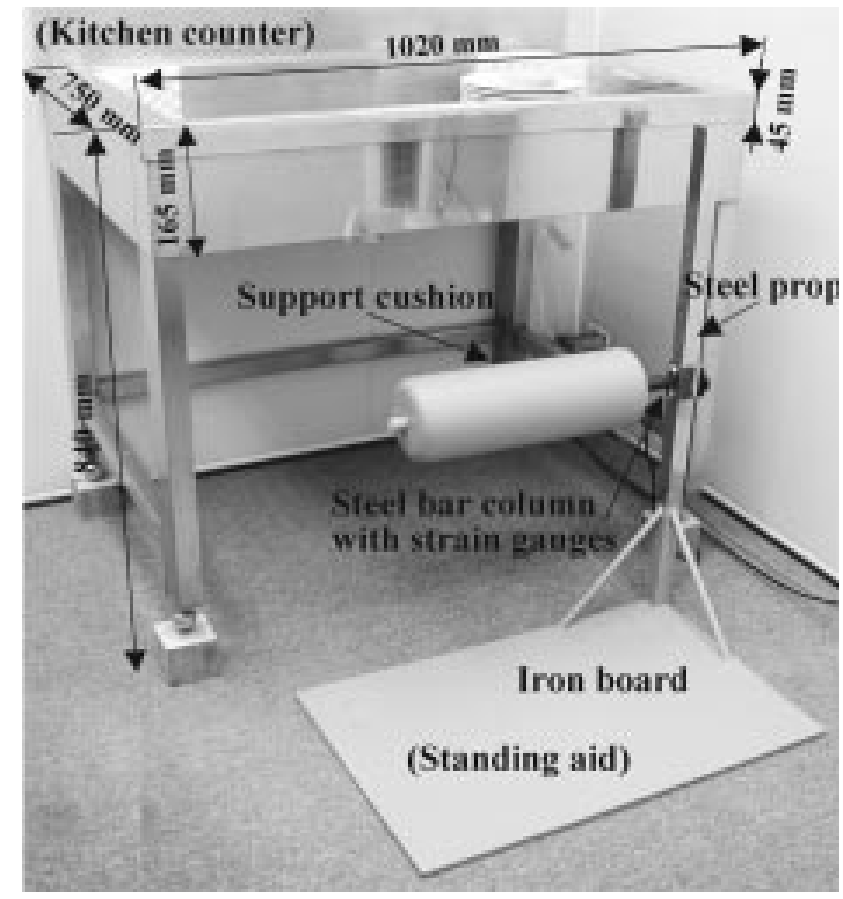

Fig. 1. Standing aid with a round type of cushion and a kitchen counter.

adjusted in multiples of $25 \mathrm{~mm}$ and the angle could be adjusted continuously. The right hand of the steel bar was connected to the steel prop. Eight strain gauges were attached to the connection end of the steel bar to measure the horizontal and the vertical strain forces acting on the bar. The kitchen counter (width $1020 \mathrm{~mm} \times$ length $750 \mathrm{~mm} \times$ height 840 $\mathrm{mm}$ ) was made of stainless steel. This height for the kitchen counter was adopted since it was the height commonly observed in facilities that we investigated. The depth of the sink in the kitchen counter was $165 \mathrm{~mm}$ and the height of the upper edge was $45 \mathrm{~mm}$. A $470 \mathrm{~g}$ plate with a diameter of $220 \mathrm{~mm}$ was used for dishwashing.

\section{Experimental conditions and task}

Figure 2 shows the experimental conditions. The subjects were asked to wash the plates in each of four working postures: (a) without the standing aid (Free), (b) with the round type of aid (Round), (c) with the small rectangular type of aid (Small rectangle), and (d) with the large rectangular type of aid (Large rectangle). The subjects were instructed to support the shins with the standing aid and support the abdomen with the edge of the kitchen counter. The height of the standing aid was set at the shins since in the previous study ${ }^{13)}$ the muscle loads on the low back and legs were decreased by using the standing aid to support the shins. About the position of the standing aid at the shins, 


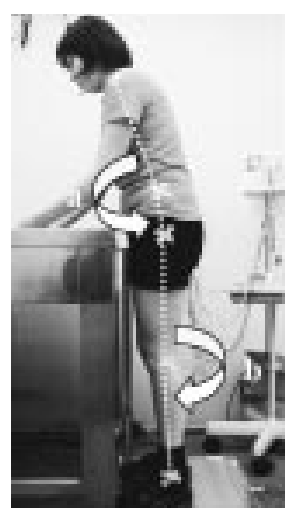

Free

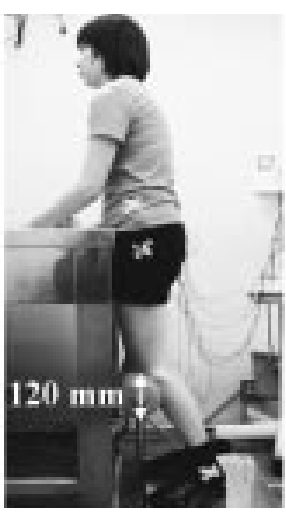

Round
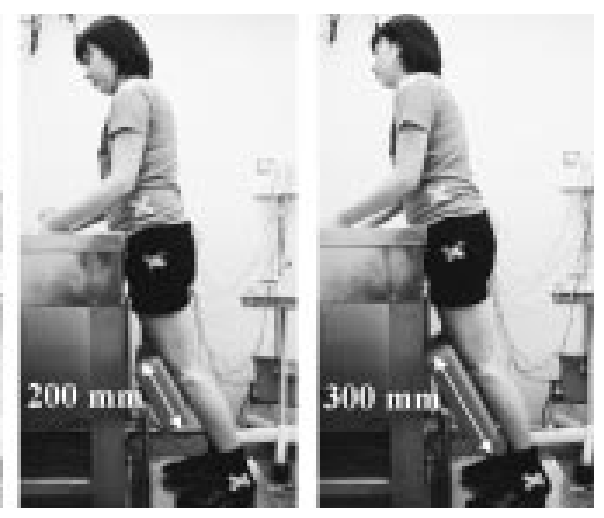

Small rectangle Large rectangle

Fig. 2. Four experimental conditions.

Free: a posture without the standing aid. Round, Small and Large rectangles: a posture with the round type, the small rectangular type and the large rectangular type of aid for supporting the shins, respectively. ${ }^{a}$ Bending angle of the trunk. ${ }^{\mathrm{b}}$ Crooking angle of the knee.

the subjects were instructed to use the round type of aid for the upper part of the shins, the small rectangular type of aid for the upper half of the shins, and the large rectangular type of aid for the whole shins. The distance between the cushion and the kitchen counter and between the feet and the kitchen counter were adjusted to the required position. In each experimental condition, the subjects were asked to stand upright without the standing aid for a minute, and then to wash the plates for $15 \mathrm{~min}$ at the rate of twenty plates per minute. In the washing task, the subjects were instructed to take the plate underneath the sink, wash it in the sink, and put it back underneath the sink. The order of the experimental conditions was completely randomized for each subject.

\section{Measurement}

The electromyogram (EMG), electrocardiogram (ECG), the strain force on the steel bar and the ground reaction force were recorded for a minute in the upright posture before the task and then recorded for 15 min during the task. The bending angle of the trunk and the crooking angle of the knee were measured for a minute in the upright posture before the task and measured every five minutes during the task. Local discomfort was rated after the task. The EMG was measured by using surface electrodes $(\mathrm{Ag}-\mathrm{AgCl})$ from six muscles: the right and left erector spinae muscles, the right and left biceps femoris muscles, and the right and left gastrocnemius muscles. The electrodes on the erector spinae muscles were positioned laterally $40 \mathrm{~mm}$ from the median line of the back at L3/L4. The electrodes for the other muscles were placed on the skin overlying the muscles. The interelectrode distance was approximately $20 \mathrm{~mm}$ and the skin impedance was below $10 \mathrm{k} \Omega$. The ECG was measured from the CM5 lead which was a bipolar lead with manubrium $(-)$ and V5 position (+) in a precordial lead. The time constant and high frequency cut-off for the EMG and ECG were set at $0.03 \mathrm{~s}$ and $300 \mathrm{~Hz}$, respectively. The EMG and ECG signals were amplified (EEG-1100, Nihon Kohden, Japan), converted analog to digital at $1,000 \mathrm{~Hz}$, and stored in a personal computer. The EMG signals were rectified and integrated every five minutes for $15 \mathrm{~min}$, and then standardized as percentages of the maximum voluntary contraction (\%MVC). The MVC of the erector spinae muscle was measured in a standing posture with a back strength dynamometer (T.K.K.5102, TAKEI, Japan). The subjects were asked to pull upward while gripping the dynamometer for $10 \mathrm{~s}$ with all their strength using the muscles of the low back. The MVC of the biceps femoris muscle was measured while standing on one leg. The subjects were asked to pull a wire ring fixed to the floor upward by a heel for $10 \mathrm{~s}$ with all their strength using the muscle of the back of the thigh. The MVC of the gastrocnemius muscle was measured while standing on tiptoe. The subjects were asked to raise the heel for $10 \mathrm{~s}$ with all their strength using the calf muscles. In this posture, the shoulder of the subject was held by an experimenter so that the heel could not be raised. All measurements of the MVC were performed at the end of the experiment. Heart rate data were calculated from the R-wave of the ECG signals every five minutes, and then standardized as a percentage of the heart rate before the task. The ground reaction force data in three axes ( $\mathrm{x}, \mathrm{y}$ and $\mathrm{z}$ ) 
were measured by means of a force plate (9286A, KISTLER, Switzerland), and then standardized as a percentage of the subject's weight. The $\mathrm{x}$-axis was the subject's right (-) and left $(+)$ directions, and the y-axis was the subject's front $(+)$ and back (-) directions, and the $\mathrm{z}$-axis was the subject's vertical (+) direction. The strain signals of the steel bar in the horizontal (Y-axis) and the vertical (Z-axis) directions were measured and subsequently amplified (AP-621G, Nihon Kohden, Japan), and stored in the computer. The data for the horizontal and the vertical strain forces of the bar were calculated, based on the strain on the weight of $0 \mathrm{~kg}, 5 \mathrm{~kg}$ and $10 \mathrm{~kg}$. The horizontal and vertical strain force data were standardized as percentages of the subject's weight. By using the vertical ground reaction force (A), the vertical strain force of the bar (B) and the subject's weight (W), the vertical force for the kitchen counter (C) was calculated with the following equation:

$$
\mathrm{C}=\mathrm{W}-|\mathrm{A}|-|\mathrm{B}|
$$

And the sum of the strain force of the bar (B) and the vertical force of the kitchen counter $(\mathrm{C})$ was defined as the decreased force by using the standing aid and the kitchen counter (D):

$$
\mathrm{D}=|\mathrm{B}|+|\mathrm{C}|
$$

The bending angle of the trunk and the crooking angle of the knee were measured by means of frame processing with photographs. The frame processing was the same as that in our previous study ${ }^{13}$. The bending angle of the trunk was formed by the acromion, iliocristale and trochanterion (Fig. 2 , a). The crooking angle of the knee was defined by the trochanterion, caput fibulae point and malleolus fibulae point (Fig. 2, b). The data for the bending angle of the trunk and the crooking angle of the knee were calculated every five minutes by subtracting the angles in the forward bending posture from the angles on the upright posture. Subjective discomfort was rated by means of a questionnaire with a 10 $\mathrm{cm}$ visual analog scale (VAS). The two ends of the scale were defined as "nothing at all $(0 \mathrm{~cm})$ " and "extreme discomfort $(10 \mathrm{~cm})$ ". The subjects were instructed to evaluate the discomfort in body regions during the task and then to enter the rating points. The body regions were as follows: neck, shoulder, arm, hand, upper back, lower back, front of thigh, back of thigh, knee, shin, calf, tiptoe and heel. These regions were selected according to the chart used for identification of body parts by Corlett and Bishop ${ }^{14)}$ and Van Dieën et $a l .{ }^{15)}$. The rating points were standardized as the ratio of discomfort in the posture without the standing aid (Free).

\section{Statistical analysis}

The data for the rating of local discomfort and the position of the bar were subjected to statistical analysis with one way repeated-measures ANOVA. All data except for the discomfort and bar data were subjected to statistical analysis with two way repeated-measures ANOVA. Statistically significant results of the ANOVAs were subjected to multiple comparisons with Tukey's test to determine the differences in the parameters for posture conditions and working time. For each posture condition, correlation analysis was done to determine the relationship of the bending angle of the trunk, the rating of local discomfort (the upper and lower back), and the \%MVC (the right and left erector spinae muscles) with each subject's height. Statistical significance was set at $p<0.05$.

\section{Results}

\section{Working posture}

The bending angle of the trunk $(F=13.80, d f=3,24$, $P<0.001)$ and the crooking angle of the knee $(F=4.27$, $d f=3,24, P=0.047$ ) were significantly affected by the posture conditions (Table 1). The bending angle of the trunk without the aid (Free) was significantly larger than those with the three types of aid. The crooking angle of the knee with the round type of aid (Round) was significantly larger than those without the aid (Free) or with the large rectangular type of aid (Large rectangle). The ground reaction forces in the vertical direction $(F=16.21, d f=3,24, P<0.001)$, the front and back directions ( $F=5.47, d f=3,24, P=0.005)$, and the right and left directions $(F=208.37, d f=3,24, P<0.001)$ were significantly affected by the posture conditions (Table 1 ). The vertical and backward ground reaction forces without the aid (Free) were significantly larger than those with the three types of aid. The leftward ground reaction forces with both the rectangular types of aid (Small and Large rectangles) were significantly larger than without the aid (Free). The ground reaction force in the vertical direction was significantly affected by working time $(F=11.32, d f=2,16$, $P=0.007)$. The vertical ground reaction force was the largest in the first session from 0 to $5 \min (85.4 \pm 11.7 \%)$, followed by the second session from 5 to $10 \mathrm{~min}(83.3 \pm 13.7 \%)$ and the third session from 10 to $15 \mathrm{~min}(81.8 \pm 14.4 \%)$. The strain force of the bar in the vertical direction $(F=4.78$, $d f=3,24, P=0.02$ ) was significantly affected by the posture conditions (Table 1). The vertical strain force of the bar with the round type of aid (Round) was significantly larger than that with the large rectangular type of aid (Large rectangle). The strain force of the bar in the horizontal 
Table 1. Results of measurements for the bending angle of the trunk, the crooking angle of the knee, the ground reaction force, the strain force of the bar, the position of the bar and heart rate

\begin{tabular}{|c|c|c|c|c|c|}
\hline & Free $^{a}$ & Round $^{\mathrm{b}}$ & $\begin{array}{c}\text { Small } \\
\text { rectangle }^{\mathrm{b}}\end{array}$ & $\begin{array}{c}\text { Large } \\
\text { rectangle }^{\mathrm{b}}\end{array}$ & $\begin{array}{c}\text { Multiple } \\
\text { comparison }^{c}\end{array}$ \\
\hline Bending angle of the trunk (degree) & $11.2 \pm 7.8$ & $1.8 \pm 7.0$ & $1.9 \pm 6.4$ & $1.4 \pm 7.4$ & $\begin{array}{l}\text { Free }>\text { Round } \\
\text { Free }>\text { Small rectangle } \\
\text { Free }>\text { Large rectangle }\end{array}$ \\
\hline Crooking angle of the knee (degree) & $6.9 \pm 11.0$ & $18.9 \pm 17.0$ & $9.2 \pm 17.1$ & $7.2 \pm 15.1$ & $\begin{array}{l}\text { Round }>\text { Free } \\
\text { Round }>\text { Large rectangle }\end{array}$ \\
\hline \multicolumn{6}{|l|}{ Ground reaction force $(\%)^{\mathrm{d}}$} \\
\hline Z-axix: downword (+) & $98.6 \pm 2.0$ & $77.3 \pm 14.6$ & $78.6 \pm 8.2$ & $79.4 \pm 11.2$ & $\begin{array}{l}\text { Free }>\text { Round } \\
\text { Free }>\text { Small rectangle } \\
\text { Free }>\text { Large rectangle }\end{array}$ \\
\hline Y-axix: forward (+) and backword (-) & $-0.6 \pm 0.7$ & $-26.0 \pm 4.7$ & $-29.3 \pm 1.6$ & $-27.8 \pm 3.9$ & $\begin{array}{l}\text { Free }>\text { Round } \\
\text { Free }>\text { Small rectangle } \\
\text { Free }>\text { Large rectangle }\end{array}$ \\
\hline $\mathrm{X}$-axix: leftward (+) and rightward (-) & $0.4 \pm 0.7$ & $0.8 \pm 1.5$ & $2.2 \pm 1.8$ & $2.1 \pm 1.5$ & $\begin{array}{l}\text { Small rectangle }>\text { Free } \\
\text { Large rectangle }>\text { Free }\end{array}$ \\
\hline \multicolumn{6}{|l|}{ Strain force of the bar $(\%)^{d}$} \\
\hline $\begin{array}{l}\text { Vertical direction (Z-axix): } \\
\text { downward (+) and upward (-) }\end{array}$ & & $10.8 \pm 4.7$ & $8.4 \pm 3.0$ & $7.7 \pm 3.2$ & Round $>$ Large rectangle \\
\hline $\begin{array}{l}\text { Horizontal direction (Y-axix): } \\
\text { forward (+) and backward (-) }\end{array}$ & & $26.7 \pm 5.3$ & $22.6 \pm 4.3$ & $24.4 \pm 5.7$ & ns \\
\hline \multicolumn{6}{|l|}{ Position of the bar (mm) } \\
\hline Height of the bar & & $375 \pm 25$ & $336 \pm 22$ & $325 \pm 22$ & $\begin{array}{l}\text { Round }>\text { Small rectangle } \\
\text { Round }>\text { Large rectangle }\end{array}$ \\
\hline Distance from the kitchen counter to the bar & & $54 \pm 13$ & $49 \pm 15$ & $41 \pm 13$ & ns \\
\hline Heart rate $(\%)^{\mathrm{e}}$ & $112.7 \pm 4.0$ & $112.6 \pm 2.1$ & $112.8 \pm 6.1$ & $115.0 \pm 6.4$ & $\mathrm{~ns}$ \\
\hline
\end{tabular}

Data are means $\pm \mathrm{SD}$ for nine subjects for $15 \mathrm{~min} .{ }^{a}$ : A posture without the standing aid. ${ }^{\text {b}}$ : A posture with the round type, the small rectangular type and the large rectangular type of aid for supporting the shins, respectively. ${ }^{c}$ : Tukey's multiple comparison test; $p<0.05$, ns: non-significant. d: The proportion was calculated by dividing the ground reaction force $(\mathrm{N})$ or the strain force of the bar $(\mathrm{N})$ by the subject's weight $(\mathrm{N})$. e : The proportion was calculated by dividing the heart rate (beats/min) during task by baseline rate before task.

direction was significantly affected by working time $(F=11.15, d f=2,16, P=0.001)$. The horizontal strain force of the bar was the largest in the first session from 0 to $5 \mathrm{~min}$ $(28.0 \pm 5.7 \%)$, followed by the second session from 5 to 10 $\min (25.7 \pm 5.1 \%)$ and the third session from 10 to $15 \mathrm{~min}$ $(24.0 \pm 5.4 \%)$. The horizontal strain force in the first session from 0 to $5 \mathrm{~min}$ was significantly larger than that in the third session from 10 to $15 \mathrm{~min}(P=0.001)$. The height of the bar $(F=38.29, d f=2,16, P<0.001)$ was significantly affected by the posture conditions (Table 1 ). The height of the bar with the round type of aid (Round) was significantly higher than that with both the rectangular types of aid (Small and Large rectangles). No effect of the posture conditions was significant for the distance from the kitchen counter to the $\operatorname{bar}(F=2.67, d f=2,16, P=0.10)$.
Relative share of vertical force for supporting the body

On average, the standing aid supported $9.0 \pm 3.2 \%$ (B) of the weight and the kitchen counter supported $12.6 \pm 7.5 \%$ (C) of the weight (Table 2). The decrease in vertical force (D) due to the combination of the standing aid and the kitchen counter was $21.5 \pm 10.0 \%$ of the weight. Without the standing aid (Free), the decrease was $1.4 \pm 2.0 \%$.

\section{Local discomfort}

The ratings of discomfort were relatively high at the upper back $(4.2 \pm 3.3 \mathrm{~cm})$ and the lower back $(5.5 \pm 3.2 \mathrm{~cm})$ in the posture without the standing aid (Free). The ratings in the upper back $(F=7.08, d f=3,24, P=0.001)$ and the lower back $(F=14.65, d f=3,24, P<0.001)$ were significantly affected by the posture conditions (Table 3 ). In both the regions, the ratings with the three types of aid were significantly lower than that without the standing aid (Free). No significant 
Table 2. The ratios of vertical ground reaction force, vertical strain force of the bar and vertical force of the kitchen counter to the subject's weight

\begin{tabular}{|c|c|c|c|c|c|}
\hline In the vertical direction & Free $^{a}$ & Round $^{\mathrm{b}}$ & $\begin{array}{c}\text { Small } \\
\text { rectangle }^{\mathrm{b}}\end{array}$ & $\begin{array}{c}\text { Large } \\
\text { rectangle }^{\mathrm{b}}\end{array}$ & $\begin{array}{c}\text { Mean values for } \\
\text { three types }\end{array}$ \\
\hline W: weight $(\%)$ & 100 & 100 & 100 & 100 & 100 \\
\hline A: ground reaction force $(\%)$ & $98.6 \pm 2.0$ & $77.3 \pm 14.6$ & $78.6 \pm 8.2$ & $79.4 \pm 11.2$ & $78.5 \pm 10.0$ \\
\hline B: strain force of the bar $(\%)$ & 0.0 & $10.8 \pm 4.7$ & $8.4 \pm 3.0$ & $7.7 \pm 3.2$ & $9.0 \pm 3.2$ \\
\hline $\mathrm{C}: \mathrm{W}-|\mathrm{A}|-|\mathrm{B}|$; vertical force of the kitchen counter $(\%)$ & $1.4 \pm 2.0$ & $11.9 \pm 11.6$ & $13.0 \pm 6.7$ & $12.9 \pm 8.8$ & $12.6 \pm 7.5$ \\
\hline $\mathrm{D}:|\mathrm{B}|+|\mathrm{C}|(\%)$ & $1.4 \pm 2.0$ & $22.7 \pm 14.6$ & $21.4 \pm 8.2$ & $20.6 \pm 11.2$ & $21.5 \pm 10.0$ \\
\hline
\end{tabular}

Data are means $\pm \mathrm{SD}$ for nine subjects for $15 \mathrm{~min} .{ }^{\text {a: }}$ A posture without the standing aid. ${ }^{\mathrm{b}}$ : A posture with the round type, the small rectangular type and the large rectangular type of aid for supporting the shins, respectively. ${ }^{c}$ : Average data for three types of aid.

Table 3. The ratio of the rating of local discomfort in the four working postures to that in the posture without the standing aid

\begin{tabular}{|c|c|c|c|c|c|}
\hline Region & $\begin{array}{c}\text { Free }^{\mathrm{a}}(10 \mathrm{~cm} \\
\text { visual analog scale })\end{array}$ & Round $^{\mathrm{b}}$ & $\begin{array}{c}\text { Small } \\
\text { rectangle }^{\mathrm{b}}\end{array}$ & $\begin{array}{c}\text { Large } \\
\text { rectangle }^{\mathrm{b}}\end{array}$ & $\begin{array}{c}\text { Multiple } \\
\text { comparison }^{\mathrm{c}}\end{array}$ \\
\hline Neck & $1.0(1.4 \pm 2.9 \mathrm{~cm})$ & $0.8 \pm 0.3$ & $0.9 \pm 0.6$ & $0.7 \pm 0.4$ & ns \\
\hline Shoulder & $1.0(1.9 \pm 3.1 \mathrm{~cm})$ & $0.7 \pm 0.3$ & $0.9 \pm 0.5$ & $0.8 \pm 0.6$ & ns \\
\hline Arm & $1.0(2.4 \pm 3.2 \mathrm{~cm})$ & $0.9 \pm 0.9$ & $1.0 \pm 0.9$ & $1.0 \pm 1.0$ & ns \\
\hline Hand & $1.0(2.6 \pm 3.1 \mathrm{~cm})$ & $1.2 \pm 0.9$ & $1.1 \pm 0.9$ & $1.1 \pm 0.7$ & ns \\
\hline Upper back & $1.0(4.2 \pm 3.3 \mathrm{~cm})$ & $0.4 \pm 0.3$ & $0.6 \pm 0.3$ & $0.6 \pm 0.5$ & $\begin{array}{l}\text { Free }>\text { Round } \\
\text { Free }>\text { Small rectangle } \\
\text { Free }>\text { Large rectangle }\end{array}$ \\
\hline Lower back & $1.0(5.5 \pm 3.2 \mathrm{~cm})$ & $0.4 \pm 0.3$ & $0.5 \pm 0.2$ & $0.6 \pm 0.3$ & $\begin{array}{l}\text { Free }>\text { Round } \\
\text { Free }>\text { Small rectangle } \\
\text { Free }>\text { Large rectangle }\end{array}$ \\
\hline Front of thigh & $1.0(1.5 \pm 1.5 \mathrm{~cm})$ & $0.9 \pm 0.3$ & $1.0 \pm 0.4$ & $1.1 \pm 0.5$ & ns \\
\hline Back of thigh & $1.0(2.3 \pm 2.1 \mathrm{~cm})$ & $0.8 \pm 0.2$ & $1.1 \pm 0.6$ & $2.0 \pm 2.5$ & $\mathrm{~ns}$ \\
\hline Knee & $1.0(1.0 \pm 1.4 \mathrm{~cm})$ & $1.6 \pm 0.9$ & $2.1 \pm 1.4$ & $2.8 \pm 4.4$ & $\mathrm{~ns}$ \\
\hline Shin & $1.0(1.2 \pm 1.4 \mathrm{~cm})$ & $0.8 \pm 0.6$ & $2.8 \pm 5.0$ & $1.2 \pm 1.0$ & ns \\
\hline Calf & $1.0(2.7 \pm 2.3 \mathrm{~cm})$ & $0.8 \pm 0.6$ & $2.1 \pm 3.0$ & $2.0 \pm 2.6$ & $\mathrm{~ns}$ \\
\hline Tiptoe & $1.0(2.5 \pm 2.3 \mathrm{~cm})$ & $0.7 \pm 0.4$ & $0.9 \pm 0.7$ & $2.3 \pm 4.8$ & ns \\
\hline Heel & $1.0(2.0 \pm 1.8 \mathrm{~cm})$ & $1.9 \pm 1.5$ & $2.6 \pm 2.2$ & $2.5 \pm 2.4$ & $\mathrm{~ns}$ \\
\hline
\end{tabular}

Data are means \pm SD for nine subjects. ${ }^{a}$ : A posture without the standing aid. ${ }^{b}$ : A posture with the round type, the small rectangular type and the large rectangular type of aid for supporting the shins, respectively. ': Tukey's multiple comparison test; $p<0.05$, ns: non-significant.

effect was found in the other body regions. With both the rectangular types of aid, however, the mean ratings of discomfort exceeded 2.0 for some regions in the lower half of the body.

\section{$E M G$ and heart rate}

The right and left erector spinae muscles (right: $F=11.81$, $d f=3,24, P<0.001$, left: $F=3.36, d f=3,24, P=0.04)$ and the right gastrocnemius muscle $(F=16.12, d f=3,24, P<0.001)$ were significantly affected by the posture conditions (Fig. 3). For the right erector spinae muscle and the right gastrocnemius muscle, the muscle activity was significantly smaller in the postures with the three types of aid than in the posture without the aid (Free). For the left erector spinae muscle, the muscle activity was significantly smaller in the posture with the round type of aid (Round) than without the aid (Free).

Mean heart rate with SD in the upright posture before the task was $83 \pm 9$ beats/min. When the ratio of heart rate during the task to that before the task was calculated, neither the effect of the posture conditions $(F=0.85, d f=3,24, P=0.48$ ) nor interaction $(F=0.44, d f=6,48, P=0.85)$ was significant (Table 1$)$. The heart rate was significantly affected by working time $(F=25.68, d f=2,16, P<0.001)$. The heart rate was the greatest in the third session from 10 to $15 \mathrm{~min}$ (115.8 $\pm 5.5 \%$ ), followed by the second session from 5 to $10 \mathrm{~min}$ 
Left erector spinae muscle

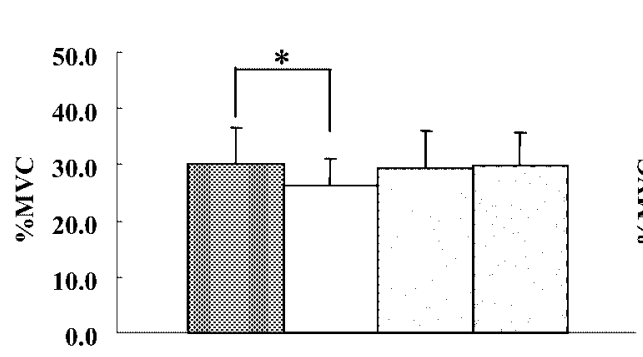

Left biceps femoris muscle

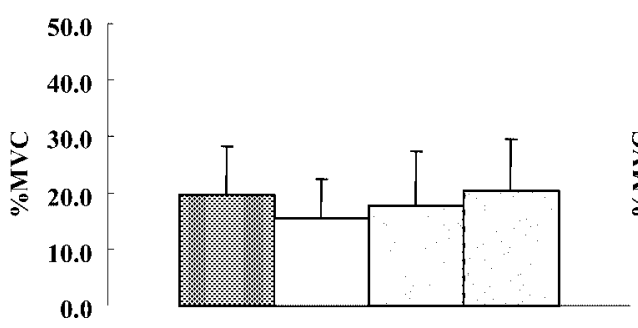

Left gastrocnemius muscle

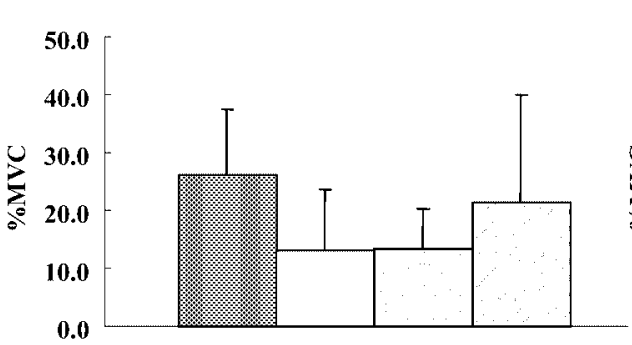

Right erector spinae muscle

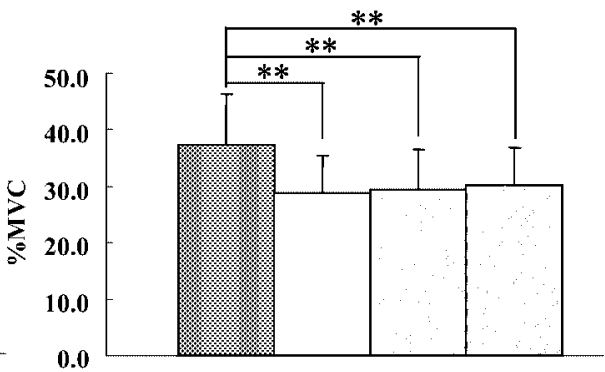

Right biceps femoris muscle

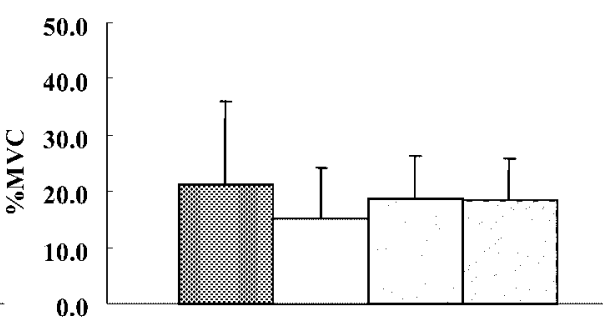

Right gastrocnemius muscle

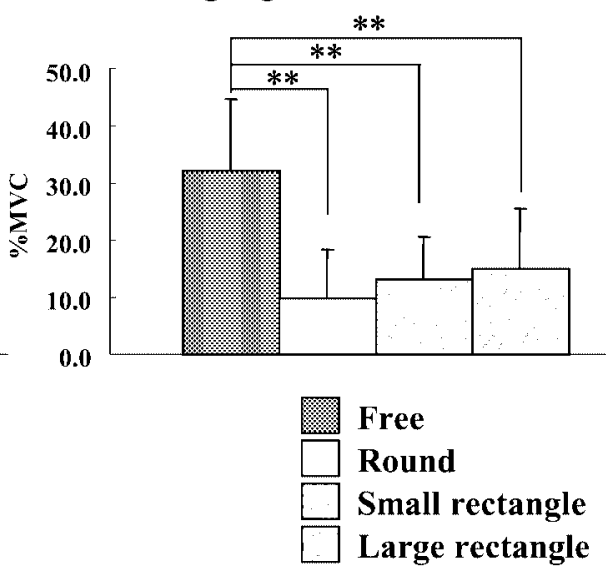

Fig. 3. Percentage of maximum voluntary contraction (\% MVC) during task in six muscles.

Data are means \pm SD for nine subjects for $15 \mathrm{~min}$. Free: a posture without the standing aid. Round, Small and Large rectangles: a posture with the round type, the small rectangular type and the large rectangular type of aid for supporting the shins, respectively. *: $p<0.05$, **: $p<0.01$ by Tukey's multiple comparison test.

$(113.7 \pm 5.2 \%)$ and the first session from 0 to $5 \min (110.3$ $\pm 4.9 \%)$. The heart rates in the sessions from 10 to $15 \mathrm{~min}$ and from 5 to $10 \mathrm{~min}$ were significantly larger than from 0 to $5 \mathrm{~min}$ ( $P=0.003$ and $P<0.001$, respectively).

Relationship between the subject's height and the low back load

With the large rectangular type of aid (Large rectangle), a significant positive correlation was observed between the height of the subject and \%MVC in the right erector spinae muscle (Table 4). Without the standing aid (Free) and with the small rectangular type of aid (Small rectangle), a significant negative correlation was observed between the height and \%MVC in the left erector spinae muscle. With the round type of aid, no significant correlation was found between the height and each parameter.

\section{Discussion}

This study suggested that supporting the body on the 
Table 4. Correlations of subject's height with the bending angle of the trunk, the rating of local discomfort (the upper and the lower back), and \% MVC (the right and left erector spinae muscles)

\begin{tabular}{lcccc}
\hline \multicolumn{1}{c}{ Parameters } & Free $^{\mathrm{a}}$ & Round $^{\mathrm{b}}$ & Small rectangle $^{\mathrm{b}}$ & Large rectangle $^{\mathrm{b}}$ \\
\hline Bending angle of the trunk & -0.03 & -0.25 & -0.15 & -0.12 \\
& $(0.87)$ & $(0.21)$ & $(0.46)$ & $(0.55)$ \\
Rating of local discomfort in the upper back & $\mathrm{c}$ & -0.45 & -0.18 & -0.46 \\
& & $(0.22)$ & $(0.65)$ & $(0.22)$ \\
Rating of local discomfort in the lower back & $\mathrm{c}$ & 0.25 & 0.19 & 0.65 \\
& & $(0.53)$ & $(0.63)$ & $(0.06)$ \\
\%MVC in the right erector spinae muscle & -0.13 & 0.29 & 0.19 & 0.38 \\
& $(0.52)$ & $(0.14)$ & $(0.34)$ & $(0.048)$ \\
& -0.56 & -0.15 & -0.58 & -0.08 \\
& $(0.003)$ & $(0.47)$ & $(0.002)$ & $(0.69)$ \\
\hline
\end{tabular}

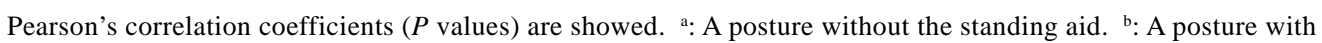
the round type, the small rectangular type and the large rectangular type of aid for supporting the shins, respectively. c: Correlation coefficient could not be analyzed because the rating points of local discomfort were standardized as ratios of measurements in the posture without the standing aid (Free).

standing aid and the kitchen counter decreased the subjective discomfort and the muscle load in the low back. The subjective discomforts and the muscle activities in the low back were smaller in the three postures with the standing aid than in the posture without the aid. Without the standing aid, the subjects did not touch the kitchen counter and the trunk was extremely bent. On the other hand, with the three types of aid, the body leaning on the standing aid and the kitchen counter was observed in all subjects. In the three postures with the standing aid, the bending angle of the trunk was smaller than that without the aid. Since the body was supported with the standing aid and the kitchen counter in the three postures, the vertical ground reaction force decreased to $78.5 \pm 10.0 \%$ of the weight. On the other hand, the vertical ground reaction force without the standing aid was almost the same as the weight. Therefore, it was suggested that the standing aid had the desired effect of decreasing discomfort and muscle load on the low back during dishwashing.

With the standing aid for supporting the shins, the ankle was extremely bent and the backward ground reaction force was larger than that without the aid, so that it would be supposed that the loads on the ankles and the calves were larger in the three postures with the standing aid than that in the posture without the aid. Nevertheless, a muscle activity in the right calf was smaller in the three postures with the standing aid than in the posture without the aid. Although heel and the calf discomfort with both the rectangular types of aid tended to be greater than that without the standing aid, no significant effect was observed in the various posture conditions. These findings were the same as those in our previous study ${ }^{13)}$ in which the working time was set at 60 min. Therefore, it was thought that the loads on the heels and the calves were not excessive when using the standing aid for up to $60 \mathrm{~min}$.

In the three postures with the standing aid, $21.5 \pm 10.0 \%$ of the weight was distributed to the aid and the kitchen counter. In the previous study ${ }^{13)}$, the vertical force distributed to the standing aid and the kitchen counter was $10.7 \%$ of the weight. This value was calculated based on the reported data for the weight and vertical ground reaction force in the previous study. The sponge used in this study was $30 \mathrm{~mm}$ thicker than that in the previous study. It was suggested that the workload on the low back was smaller in this study than in the previous study because the thick sponge lessened the discomfort in the shins and supported a larger force on the standing aid. Therefore, it was suggested that the discomfort in the low back was decreased by the thick cushion used.

In the left low back, the muscle activity with the round type of aid was significantly smaller than that without the aid, whereas muscle activities with both the rectangular types of aid were not significantly smaller than without the aid. The discomfort in the legs with both the rectangular types of aid trended to be greater than that without the aid. However, the discomfort in most body regions with the round type of aid trended to be less than that without the standing aid. Since there were only nine subjects in this experiment, between the types of aid there were no significant differences. With more subjects, the difference between the types of aid in the degree of discomfort might be significant. Taking these findings into consideration, it was thought that the 
round type of aid would be more effective than either of the rectangular types of aid in decreasing discomfort in many body regions and the muscle load on the low back.

In this study, taller subjects had increased muscle activities in the right low back in the posture with the large rectangular type of aid and decreased muscle activities in the left low back in the posture without the standing aid and with the small rectangular type of aid. In our previous study ${ }^{13}$, taller subjects had increased muscle activities in the left low back in both postures with and without the standing aid. The working time was set at $60 \mathrm{~min}$ in the previous study, whereas it was set at $15 \mathrm{~min}$ in this study, so that short work duration might have some association with the negative correlation between height and muscle activities in the left low back. It is necessary to study this relationship further.

\section{Acknowledgments}

We thank Prof. Akira Yasukouchi, Kyushu Institute of Design, and Dr. Sumiko Yamauchi, Nakamura Gakuen University at the time, for their helpful guidance and suggestions. Thanks are also due to all the volunteers who participated in the study.

\section{References}

1) Ministry of Labor, Japan (2001) General guidebook on industrial health 2001. 162-3, Japan Industrial Safety and Health Association, Tokyo.

2) Maeda K, Suenaga T, Churei M, Miyao M (1986) A factor-control study on disorders of the back, shoulders, neck and upper limbs of cooks. J Sci Labour 62, 43549 (in Japanese with English abstract).

3) Nagira T, Suzuki J, Oze Y, Ohara H, Aoyama H (1981) Cervicobrachial and low-back disorders among school lunch workers and nursery-school teachers in comparison with cash-register operators. J Hum Ergol 10, 117-24.

4) Onishi N, Watanabe A, Sakai K, Shindo H, Tsukazaki Y, Sato S (1988) Measures to improve workload of female school-lunch cookers. J Sci Labour 64, 10134 (in Japanese with English abstract).

5) Ono Y, Shimaoka M, Hiruta S, Takeuchi Y (1997) Low back pain among cooks in nursery schools. Ind Health 35, 194-201.

6) Oze Y (1984) Studies on health hazards among cooks providing school lunch service. Report 1 . The influence of working conditions in central and school kitchens on the prevalence of health hazards. Jpn J Ind Health 26, 414-24 (in Japanese with English abstract).

7) Pekkarinen A, Anttonen H (1988) The effect of working height on the loading of the muscular and skeletal systems in the kitchens of workplace canteens. Applied Ergonomics 19, 306-8.

8) Rudd J, Norton R, Robinson E, Coggan C (1996) Occupational groups at greatest risk of chronic back injury. J Occup health Safety-Aust NZ 12, 439-42.

9) Sakai K, Watanabe A, Onishi N, Shindo H, Temmyo Y (1993) Features and workload in hospital food service operation. J Sci Labour 69, 240-52 (in Japanese with English abstract).

10) Takahashi R, Urakabe $S$ (1987) Standing posture during the dish-washing task with or without the kitchen-wears. Jpn J Hum Posture 7, 39-47 (in Japanese with English abstract).

11) Burdorf A (1992) Exposure assessment of risk factors for disorders of the back in occupational epidemiology. Scand J Work Environ Health 18, 1-9.

12) Ministry of Labor, Japan (1997) Guidelines on worksite prevention of low back pain. Labor standards bureau notification No.547. Ind Health 35, 143-72.

13) Iwakiri K, Yamauchi S, Yasukouchi A (2002) Effects of a standing aid on loads on low back and legs during dishwashing. Ind Health 40, 198-206.

14) Corlett EN, Bishop RP (1976) A technique for assessing postural discomfort. Ergonomics 19, 175-82.

15) van Dieën JH, Jansen SMA, Housheer AF (1997) Differences in low back load between kneeling and seated working at ground level. Applied Ergonomics 28, 355-63. 